\title{
Glomus Tumor as a Rare Cause of Anterior Knee Pain
}

\author{
Georgios I. Drosos ${ }^{\mathrm{a}, \mathrm{c}}$, Athanasios Ververidis ${ }^{\mathrm{a}}$, Alexandra Giatromanolaki ${ }^{\mathrm{b}}$
}

\begin{abstract}
Anterior knee pain is caused by a variety of pathologies or anatomical abnormalities. Soft tissue tumors, although rare, can also be presented with anterior knee pain. A case of a glomus tumor located in front of the tibial tubercle, in a patient being treated for 2 years for chondromalacia of the patella is reported. A 28-year-old man presented to our hospital complaining of pain in the front of his right knee for approximately 2 years with gradually worsening over the recent 8 months. The patient has been treated for chondromalacia of the patella with non-steroidal anti-inflammatory drugs, physiotherapy and home exercises. A detailed physical examination of the knee revealed a painful soft tissue mass in the front of the tibial tubercle. Plain radiographs were normal. Ultrasonography of the right knee revealed an oval-shaped hyper/hypoechoic mass with multiple blood vessels in the surrounding area and clear margins. Magnetic resonance imaging (MRI) confirmed the presence of a small soft tissue mass with intermediate signal intensity similar to muscles on T1-weighted images and also intermediate signal intensity similar to surrounding fat on T2-weighted images. On T1 with fat saturation images the mass had high signal intensity (absence of fat). Finally there was an increased similar intensity of the mass after contrast medium administration (consisted of hypervascularity). The mass was excised, and the histology was characteristic of a glomus tumor. The patient was asymptomatic immediately after the operation. At the 2-year follow-up after the operation no recurrence was seen.
\end{abstract}

Keywords: Glomus tumor; Knee; Anterior knee pain; Benign tumor

Manuscript accepted for publication December 03, 2014

aDepartment of Orthopedic Surgery, Medical School, Democritus University of Thrace, University General Hospital of Alexandroupolis, Dragana, Alexandroupolis, Greece

bDepartment of Pathology, Democritus University of Thrace Medical School, University General Hospital of Alexandroupolis, Dragana, Alexandroupolis, Greece

${ }^{\mathrm{c} C}$ Corresponding Author: Georgios I. Drosos, Department of Orthopedic Surgery, Medical School, Democritus University of Thrace, University General Hospital of Alexandroupolis, Dragana, Alexandroupolis, Greece.

Email: drosos@otenet.gr

doi: http://dx.doi.org/10.14740/jmc2014w

\section{Introduction}

Anterior knee pain is caused by a variety of pathologies or anatomical abnormalities. Patellofemoral pain syndrome seems to be the most common cause in young patients and athletes in particular, but other intra-articular knee pathologies like plica syndrome, and a variety of periarticular pathologies such as patellar tendinopathy, peripatellar bursitis, Sinding-Larsen-Johansson's and Osgood Schlatter's lesions, and Hoffa's disease, are also presented with anterior knee pain [1-4]. Soft tissue tumors, although rare, can also present with anterior knee pain [5].

A case of a glomus tumor located in front of the tibial tubercle, in a patient being treated for 2 years for chondromalacia of the patella, is reported.

\section{Case Report}

A 28-year-old man presented to our hospital complaining of pain in the front of his right knee for approximately 2 years with gradually worsening over the recent 8 months. The patient had been treated for chondromalacia of the patella with nonsteroidal anti-inflammatory drugs, physiotherapy and home exercises. There was no history of trauma. Pain increased with movements of the right knee, such as walking or squatting, and it was relieved by resting, especially when the knee was slightly flexed.

The patient walked freely and without limping. A detailed physical examination of the knee revealed patellofemoral pain, as well as a point of tenderness on palpation localized in the front of the tibial tubercle. A soft tissue mass in front of the tibial tubercle not fixed with the surrounding tissues was found. The knee was otherwise normal and had a normal range of motion.

Plain radiographs were normal. Ultrasonography of the right knee revealed an oval-shaped hyper/hypoechoic mass with multiple blood vessels in the surrounding area and clear margins. Magnetic resonance imaging (MRI) confirmed the presence of a small soft tissue mass measured (dimensions: 24 $\times 10 \times 20 \mathrm{~mm}$ ) on the front of the tibial tubercle. The mass had intermediate signal intensity similar to muscles on T1-weighted images (Fig. 1) and also intermediate signal intensity simi- 


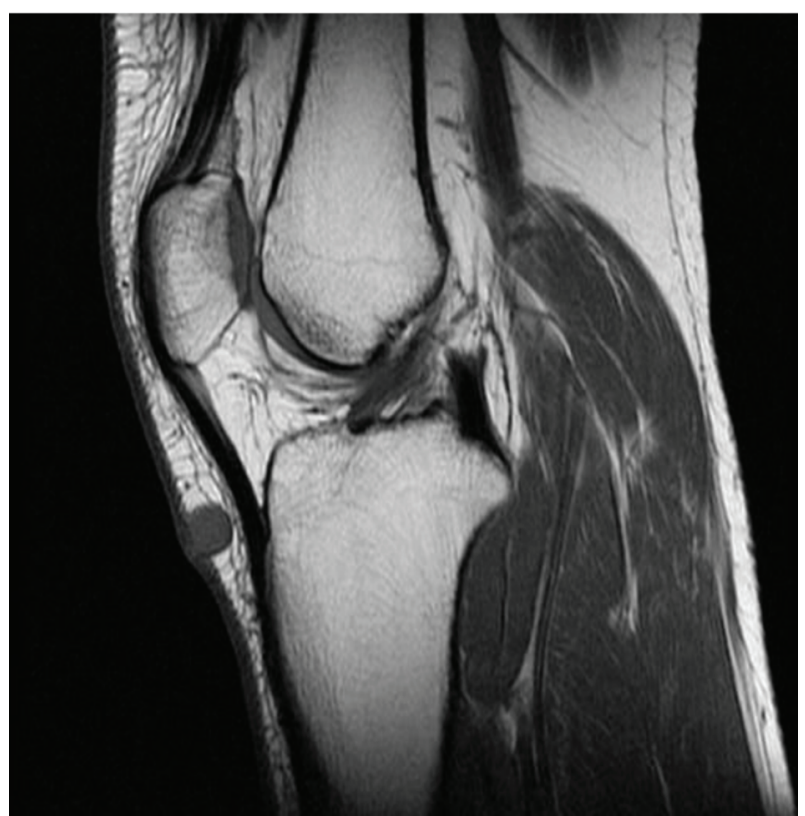

Figure 1. T1-weighted image showing a small soft tissue mass with intermediate signal intensity, similar to those of muscles.

lar to surrounding fat on T2-weighted images (Fig. 2). On T1 with fat saturation images the mass had high signal intensity (absence of fat) (Fig. 3). Finally, there was an increased similar intensity of the mass after contrast medium administration (consisted of hypervascularity).

The patient was treated with excision of the mass under local anesthesia. Macroscopically the mass was a soft, bluish colored round, and with smooth surface, $1 \mathrm{~cm}$ in diameter, with visible capillaries passing through. The patient recovered

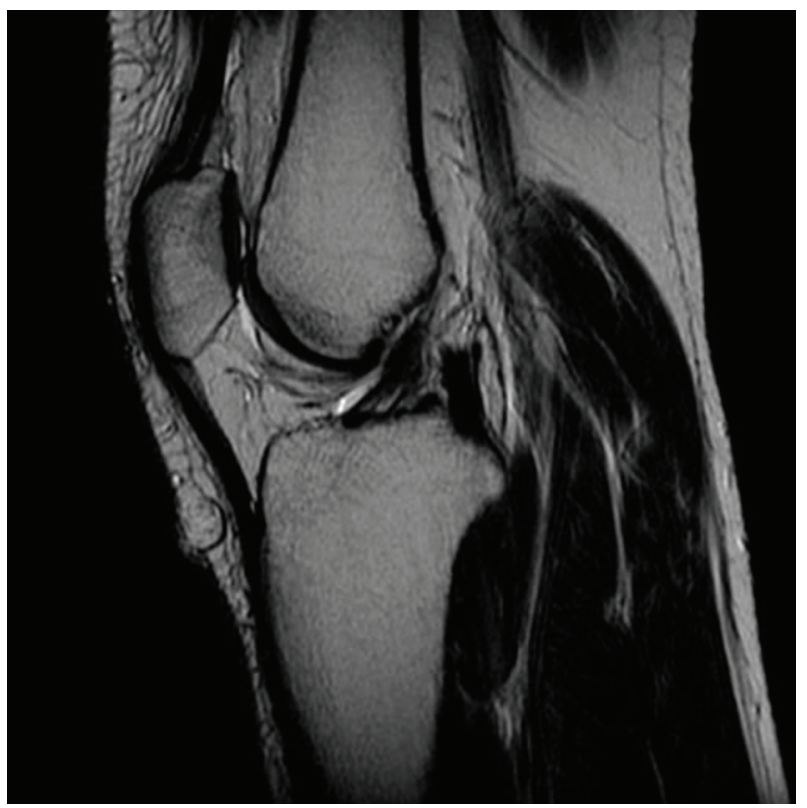

Figure 2. T2-weighted image showing a small soft tissue mass with intermediate intensity similar to the surrounding fat.

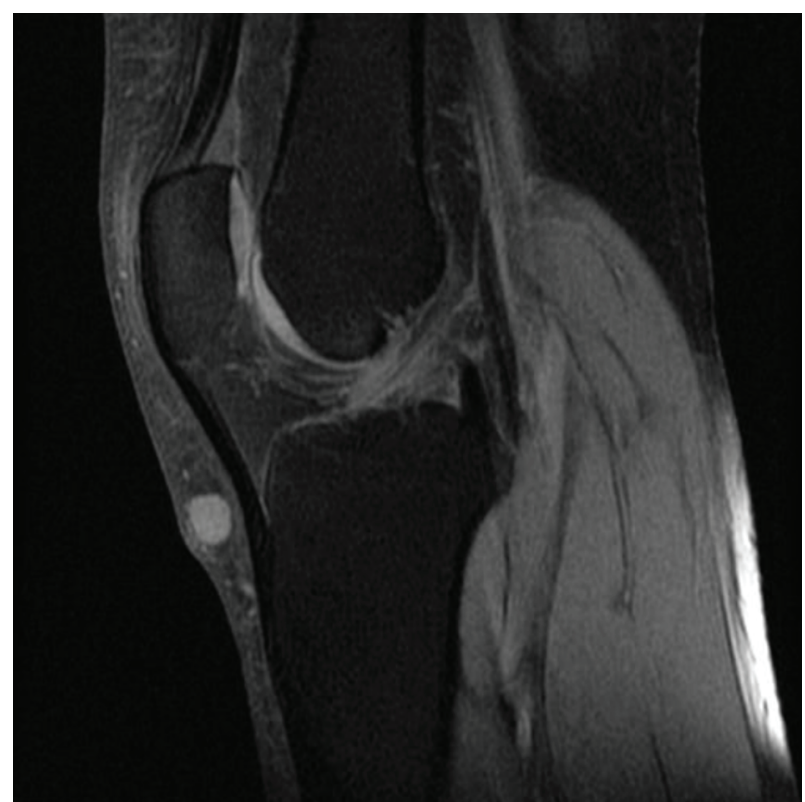

Figure 3. T1 with fat saturation image. The mass with a high signal intensity.

without complications. Immediately after operation, the pain disappeared and a few days later the patient was walking freely without crutches.

Histopathologic examination of the mass revealed a glomangioma composed of uniform round or polygonal cells with regular nuclei and pale or eosinophilic cytoplasm arranged around vascular structures (Fig. 4). The glomus ("epitheloid") cells were immunohistochemically positive for vimentin and $\alpha$-smooth muscle actin and negative for keratins and desmin.

At the time of the last follow-up, 2 years post-operatively, the patient is free of any symptoms.

\section{Discussion}

Glomus tumor, first described in detail by Masson in 1934, is a rare benign vascular tumor from the neuromyoarterial canal system called the Sucquet-Hoyer canals of the glomus body in the skin [6]. They are rare and represent less than $1.6 \%$ of all benign soft tissue neoplasms [7], usually found between ages 30 and 50 and are more common in women than men with a ratio of $2: 1$ [8]. As much as $70-75 \%$ of these tumors involve the hand [7], and their typic location is to the distal parts of the phalanges and nail beds [9].

Apart from their typical location in the subungual region, ectopic locations are not uncommon [10]. In the lower limb these lesions have been described in the ankle [11], the heel pad [12] and the knee [6, 13].

Two clinical presentations of these tumors can be indentified: solitary and multiple [10]. The etiology of a solitary glomus tumor is unknown, whereas the etiology of multiple glomus tumors is associated with an autosomal dominant heritable mutation localized to chromosome 1p21-22 [14-16].

One of the distinguishing features of glomus tumors is the 

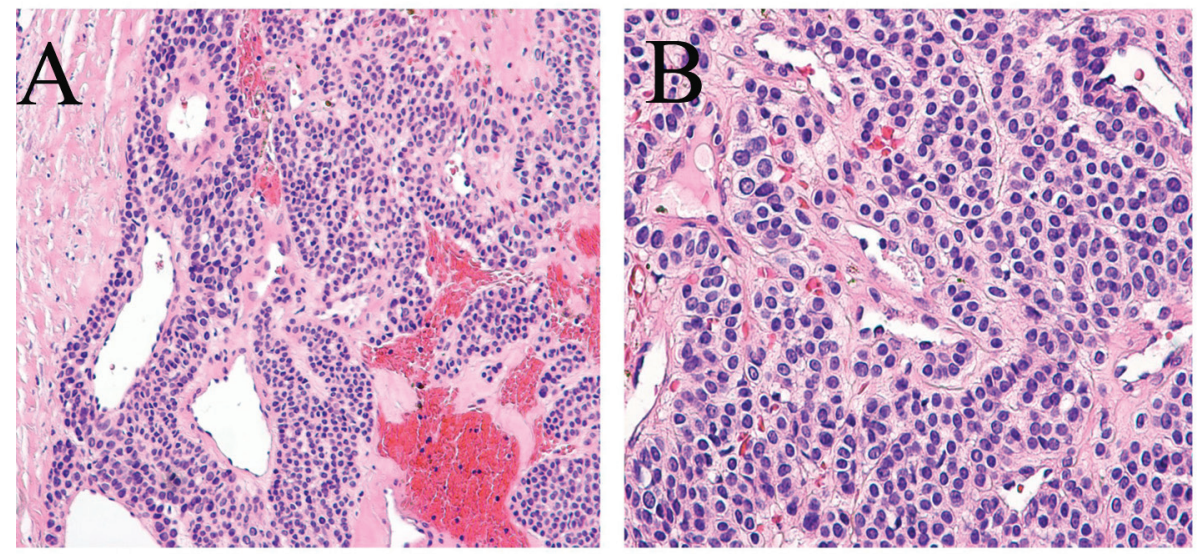

Figure 4. Histology of the mass showing uniform round or polygonal cells with regular nuclei and pale or eosinophilic cytoplasm arranged around vascular structures $(A, \times 200 ; B, \times 400)$.

presentation of the classic triad of symptoms: 1) hypersensitivity to cold, 2) paroxysmal severe pain and 3) pinpoint tenderness to blunt palpation [10]. According to the largest retrospective study by Van Geertruyden et al [9] bluish discoloration was present in $28 \%$ of patients and pulp nodule occurred in $33 \%$ of patients. Although these symptoms are quite characteristic, many patients with glomus tumors do not present with this classic triad.

There are three main clinical tests used to diagnose glomus tumors, such as the love pin test, in which positive result is the patients' experience of severe pain. The Hildreth test, involves applying a tourniquet to the base of the suspected area and repeating the love pin test. A positive result is the lack of pain in the patient [17]. The third is exposure to cold, which should produce pain related to cold sensitivity [10]. Nevertheless, these tests have limited use, as they are not $100 \%$ specific $[10,17]$.

Other diagnostic tools available include MRI (which is the most sensitive) [18] and ultrasound which is useful for the purpose of building a differential diagnosis [19].

Clinically, glomus tumors can be confused with neuromas, arthritis or gout, and differential diagnosis also includes melanoma, blue nevus and angioma [10, 20]. Misdiagnosis delays appropriate treatment. The treatment is surgical with marginal excision [21], which should result in a dramatic resolution of symptoms as occurred in our patient.

Glomus tumors around the knee have been reported in variable locations: in the lateral aspect $[20,22,23]$, in the medial aspect [13], in the popliteal fossa [24], medial to the tibial tuberosity [25], proximal and medial to the patella [25], in the patellar ligament [26], medial to the patellar ligament [23] and in front of the patellar ligament [23]. It has been also found in intra-articular but extra-synovial locations like in the fat pad [27] and beneath the plica synovialis [6].

Delay in diagnosis of glomus tumors around the knee is common and has been reported to range from 4 months to 40 years from the onset of symptoms [28]. Some of the patients do not present with the classical symptoms $[6,23]$ or with other concomitant pathologies of the knee thus delaying the diagnosis of the glomus tumor $[13,20,25,26]$.
Our patient presented with two of the classic triad signs: pain and point tenderness, but did not relay a history of cold sensitivity. It has been noted that only about $50 \%$ of patients had all components of the classic triad of signs [29]. Although there was no other knee pathology in our patient, the anterior knee pain was attributed to patellofemoral pain and missleaded the treating physician for a long time. This should be a reminder to be aware of atypical locations of glomus tumor to avoid misdiagnosis, which prolongs the serious pain of the patients.

To avoid making a wrong diagnosis, a high index of suspicion is required for the diagnosis and the MRI scanning can be helpful in investigating an unexplained knee pain or a painful lump around the knee [13]. The use of MRI to image these tumors has been described as the method of choice; however, it does not yield a detailed image of the tumor, just an adjunct to allow more precise localization [18]. Use of advanced imaging techniques is, therefore, only partly helpful and must be used in conjunction with clinical assessment of a joint, which can have a multitude of reasons for being painful [23].

In conclusion, although knee glomangioma is rare, it could be a cause of anterior knee pain.

\section{Conflict of Interest}

None.

\section{References}

1. Kannus P, Aho H, Jarvinen M, Niittymaki S. Computerized recording of visits to an outpatient sports clinic. Am J Sports Med. 1987;15(1):79-85.

2. Dixit S, DiFiori JP, Burton M, Mines B. Management of patellofemoral pain syndrome. Am Fam Physician. 2007;75(2):194-202.

3. Fulkerson JP. Diagnosis and treatment of patients with patellofemoral pain. Am J Sports Med. 2002;30(3):447456. 
4. Witvrouw E, Werner S, Mikkelsen C, Van Tiggelen D, Vanden Berghe L, Cerulli G. Clinical classification of patellofemoral pain syndrome: guidelines for non-operative treatment. Knee Surg Sports Traumatol Arthrosc. 2005;13(2):122-130.

5. Waryasz GR, McDermott AY. Patellofemoral pain syndrome (PFPS): a systematic review of anatomy and potential risk factors. Dyn Med. 2008;7:9.

6. Kato S, Fujii H, Yoshida A, Hinoki S. Glomus tumor beneath the plica synovialis in the knee: a case report. Knee. 2007;14(2):164-166.

7. Soule EH, Ghormley RK, Bulbulian AH. Scientific exhibits: primary tumors of the soft tissues of the extremities exclusive of epithelial tumors; an analysis of five hundred consecutive cases. AMA Arch Surg. 1955;70(3):462-474.

8. Diao E, Moy OJ. Common tumors. Orthop Clin North Am. 1992;23(1):187-196.

9. Van Geertruyden J, Lorea P, Goldschmidt D, de Fontaine S, Schuind F, Kinnen L, Ledoux P, et al. Glomus tumours of the hand. A retrospective study of 51 cases. J Hand Surg Br. 1996;21(2):257-260.

10. McDermott EM, Weiss AP. Glomus tumors. J Hand Surg Am. 2006;31(8):1397-1400.

11. Smyth M. Glomus-cell tumors in the lower extremity. Report of two cases. J Bone Joint Surg Am. 1971;53(1):157159.

12. Quigley JT. A glomus tumor of the heel pad. A case report. J Bone Joint Surg Am. 1979;61(3):443-444.

13. Waseem M, Jari S, Paton RW. Glomus tumour, a rare cause of knee pain: a case report. Knee. 2002;9(2):161163.

14. Hee HT, Das De S, Chong SM. Glomus tumour of the musculoskeletal system. J Orthop Surg (Hong Kong). 1997;5:105-113.

15. Boon LM, Brouillard P, Irrthum A, Karttunen L, Warman ML, Rudolph R, Mulliken JB, et al. A gene for inherited cutaneous venous anomalies ("glomangiomas") localizes to chromosome 1p21-22. Am J Hum Genet. 1999;65(1):125-133.

16. Calvert JT, Burns S, Riney TJ, Sahoo T, Orlow SJ, Nevin $\mathrm{NC}$, Haisley-Royster C, et al. Additional glomangioma families link to chromosome 1p: no evidence for genetic heterogeneity. Hum Hered. 2001;51(3):180-182.

17. Panagiotopoulos E, Maraziotis T, Karageorgos A, Dimopoulos P, Koumoundourou D. A twenty-year delay in diagnosing a glomus knee tumor. Orthopedics. 2006;29(5):451-452.

18. Matloub HS, Muoneke VN, Prevel CD, Sanger JR, Yousif NJ. Glomus tumor imaging: use of MRI for localization of occult lesions. J Hand Surg Am. 1992;17(3):472-475.

19. Fornage BD. Glomus tumors in the fingers: diagnosis with US. Radiology. 1988;167(1):183-185.

20. Okahashi K, Sugimoto K, Iwai M, Kaneko K, Samma M, Fujisawa Y, Takakura Y. Glomus tumor of the lateral aspect of the knee joint. Arch Orthop Trauma Surg. 2004;124(9):636-638.

21. Heiney JP, Leeson MC. Unique size and location of a glomus tumor with a review of the literature. J Shoulder Elbow Surg. 2009;18(1):e1-3.

22. Stambough J. Painful lateral knee mass: a report of two unusual cases. Am J Knee Surg. 1991;4:79-83.

23. Clark ML, O'Hara C, Dobson PJ, Smith AL. Glomus tumor and knee pain: a report of four cases. Knee. 2009;16(3):231-234.

24. Oztekin HH. Popliteal glomangioma mimicking Baker's cyst in a 9-year-old child: an unusual location of a glomus tumor. Arthroscopy. 2003;19(7):E19-23.

25. Puchala M, Kruczynski J, Szukalski J, Lianeri M. Glomangioma as a rare cause of knee pain. A report of two cases. J Bone Joint Surg Am. 2008;90(11):2505-2508.

26. Mabit C, Pecout C, Arnaud JP. Glomus tumor in the patellar ligament. A case report. J Bone Joint Surg Am. 1995;77(1):140-141.

27. Hardy P, Muller GP, Got C, Lortat-Jacob A, Benoit J. Glomus tumor of the fat pad. Arthroscopy. 1998;14(3):325328.

28. Pater TJ, Marks RM. Glomus tumor of the hallux: case presentation and review of the literature. Foot Ankle Int. 2004;25(6):434-437.

29. Phillips CS, Murphy MS. Vascular problems of the upper extremity: a primer for the orthopaedic surgeon. J Am Acad Orthop Surg. 2002;10(6):401-408. 